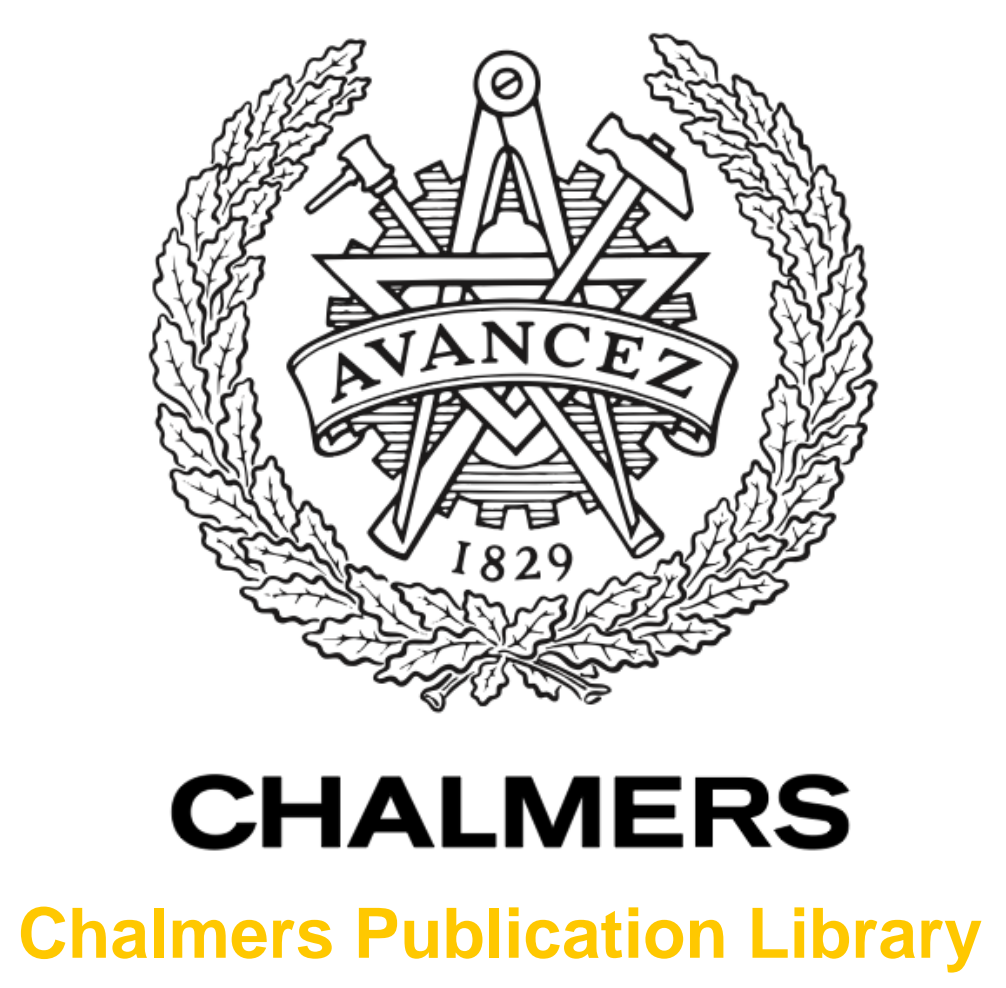

\title{
Efficient Analysis of Large Aperiodic Antenna Arrays Using the Domain Green's Function Method
}

This document has been downloaded from Chalmers Publication Library (CPL). It is the author's version of a work that was accepted for publication in:

IEEE Transactions on Antennas and Propagation (ISSN: 0018-926X)

Citation for the published paper:

Ludick, D. ; Maaskant, R. ; Davidson, D. (2014) "Efficient Analysis of Large Aperiodic Antenna Arrays Using the Domain Green's Function Method". IEEE Transactions on Antennas and Propagation, vol. 62(4), pp. 1579-1588.

http://dx.doi.org/10.1109/tap.2014.2299826

Downloaded from: http://publications.lib.chalmers.se/publication/199307

Notice: Changes introduced as a result of publishing processes such as copy-editing and formatting may not be reflected in this document. For a definitive version of this work, please refer to the published source. Please note that access to the published version might require a subscription.

Chalmers Publication Library (CPL) offers the possibility of retrieving research publications produced at Chalmers University of Technology. It covers all types of publications: articles, dissertations, licentiate theses, masters theses, conference papers, reports etc. Since 2006 it is the official tool for Chalmers official publication statistics. To ensure that Chalmers research results are disseminated as widely as possible, an Open Access Policy has been adopted.

The CPL service is administrated and maintained by Chalmers Library. 


\title{
Efficient Analysis of Large Aperiodic Antenna Arrays Using the Domain Green's Function Method
}

\author{
Daniel J. Ludick, Rob Maaskant, Senior Member, IEEE, David B. Davidson, Fellow, IEEE, \\ Ulrich Jakobus, Fellow, IEEE, Raj Mittra, Life Fellow, IEEE, and Dirk de Villiers, Member, IEEE
}

\begin{abstract}
An efficient method-of-moments (MoM) based domain decomposition technique, viz., the domain Green's function method (DGFM), is presented for analyzing large antenna arrays. The DGFM is a perturbation technique where mutual coupling between array elements is accounted for during the formulation of an active impedance matrix for each domain/array element. The active current distribution on the entire array geometry is obtained by solving the smaller matrix equations related to the elements, and not that of the problem as a whole. This leads to a significant saving in both runtime and memory usage. The method also takes into account the edge effects attributed to the finite size of the array, complex excitations with nonlinear phase shift and is not limited to periodic array configurations. The DGFM is an approximation and assumes a slowly varying current distribution between domains. A novel way to mitigate the aforementioned, by including secondary coupling effects, is also discussed. Furthermore, an efficient active impedance matrix fill strategy is presented where the active impedance matrix summation is truncated to include only a certain number of terms. Parallelization using both distributed and shared memory programming models have also been applied to the DGFM, to further optimize runtime and memory usage.
\end{abstract}

Index Terms - Domain decomposition, finite antenna arrays, method-of-moments (MoM), non-periodic arrays.

\section{INTRODUCTION}

A NALYZING large, finite, irregular-spaced antenna arrays is of interest to various research groups. One such an application for which nonuniform spaced array configurations is specifically well-suited, are for high-sensitivity imaging in the field of radio astronomy - an example being the aperture

Manuscript received December 04, 2012; revised September 09, 2013; accepted December 08, 2013. Date of publication January 13, 2014; date of current version April 03, 2014. This work was supported by the South African Research Chairs Initiative of the Department of Science and Technology and National Research Foundation and EM Software and Systems-S.A. (Pty) Ltd., as well as the Swedish VR and VINNOVA funding agencies.

D. J. Ludick, D. B. Davidson, and D. de Villiers are with the Department of Electrical and Electronic Engineering, Stellenbosch University, Stellenbosch 7600, South Africa (e-mail: dludick@emss.co.za; davidson@sun.ac.za; ddv@sun.ac.za).

U. Jakobus is with the EM Software and Systems-S.A. (Pty) Ltd, Stellenbosch 7600, South Africa (e-mail: jakobus@emss.co.za).

R. Maaskant is with the Department of Signals Systems, Chalmers University of Technology, Gothenburg S-41296, Sweden (e-mail: rob.maaskant@ chalmers.se).

R. Mittra is with the Electrical Engineering Deptartment, Pennsylvania State University, State College, PA 16801 USA (e-mail: r1mece@engr.psu.edu).

Color versions of one or more of the figures in this paper are available online at http://ieeexplore.ieee.org.

Digital Object Identifier 10.1109/TAP.2014.2299826 arrays for the low-frequency spectrum of the next-generation radio telescope, the Square Kilometre Array (SKA), viz., SKA1-low [1].

Although the SKA1-low aperture array configurations are typically sparse, with interelement spacing greater than half a wavelength, mutual coupling can still affect the performance of the array and should be accounted for in the analysis as accurately as possible. Using conventional full-wave simulation techniques, such as the method-of-moments (MoM), is typically not an option, because the arrays being considered can consist of a large number of elements. For the SKA1-low, it is estimated that the number of elements is in the order of 250000 operating over the $50-350 \mathrm{MHz}$ band [1]. Analyzing such large problems places a considerable burden on computational resources in terms of runtime and memory usage.

Various techniques for analyzing large, finite arrays have been presented over the last few decades. Small to moderate sized arrays can be analyzed using full-wave methods such as the Method-of-Moments (MoM). However, for electrically large array configurations, the MoM results in very long simulation runtimes and significant memory usage. To this end, a variety of approximation techniques have been developed to simulate large array configurations, e.g., the infinite array plus spectral windowing method [2], macro basis function techniques, such as the characteristic basis function method (CBFM, [3]), the synthetic function expansion (SFX, [4]), and the MBF method in [5], as well as iterative techniques, such as the adaptive integral method (AIM) [6] and the precorrected fast Fourier transform (pFFT) method [7].

The technique presented here for the analysis of such large, irregular arrays is the domain Green's function method (DGFM) [8], [9]. The DGFM is a perturbation technique that is fundamentally based on the work done by Skrivervik and Mosig which was developed for printed array antennas using the multi-layered Green's function [10], [11]. In the current work, the method is formulated for the scalar free-space Green's function and applied on a higher block-matrix factorization level. Mutual coupling between array elements is accounted for with the formulation of an active impedance matrix for each of the domains. ${ }^{1}$ The latter can become the dominant operation in the total solution time for very large array configurations. To overcome this limitation, the DGFM is extended by first sorting the elements according to descending mutual coupling strength in the active array environment. The sorting criteria that is used, accounts for both the complex excitation and the

\footnotetext{
${ }^{1}$ Sometimes referred to as scan impedance matrix.
} 
distance between the domains. The aforementioned is then used to truncate the summation of the active impedance matrix calculation to include only a certain number of terms.

As discussed in [10], [11], a perturbation approach such as the DGFM requires that the current distribution between the elements be slowly varying. For this very reason, the accuracy of the DGFM deteriorates when investigating off-broadside scan angles for a phased array example, as will be illustrated and discussed in Section V.

Furthermore, a novel technique is proposed for improving the accuracy of the DGFM. The technique is based on applying an approximate solution obtained iteratively with a Jacobi decomposition of the DGFM block matrix factorization [12]. The DGFM has also been integrated as part of an efficient array analysis tool in the commercial computational electromagnetics software package, FEKO [13].

This paper is organized as follows. In Section II-A, the DGFM is introduced and its mathematical formulation is presented. A comparison with macro basis function techniques, such as the CBFM [14], is presented in Section II-B. An acceleration strategy for calculating the active impedance matrices is discussed in Section III. The parallelization of the DGFM is explained in Sections IV and in V simulation results are presented for various irregular array configurations consisting of log-periodic type elements called the Zig-Zag, based on work presented in [15] and [16]. The work is then concluded in Section VI with a summary and also some recommendations for future research. A method for improving the solution accuracy of the DGFM, that is based on the Jacobi decomposition of the block factorized MoM matrix, is discussed in Appendix A.

\section{The DOMAIN GREEN’s Function Method}

\section{A. Mathematical Formulation}

Consider the simple array configuration illustrated in Fig. 1 consisting of $M$ identically discretized elements, with $N_{i}$ basis functions per element. We begin by applying the infinite array assumption as done in [10], i.e., that the unknown surface currents on all the array elements are identical to the surface current on the element that we wish to analyze, scaled by the complex array excitation coefficients. The aforementioned may be written as

$$
\mathbf{J}_{k}\left(\boldsymbol{r}+\boldsymbol{r}_{k i}\right) \simeq \frac{C_{k}}{C_{i}} \mathbf{J}_{i}(\boldsymbol{r})=\alpha_{k i} \mathbf{J}_{i}(\boldsymbol{r})
$$

where $\mathrm{J}_{k, i}$ and $C_{k, i}$ are the unknown surface currents and known complex excitation coefficients of array elements $k$ and $i$, respectively. The term $\alpha_{k i}$ is the complex weighting factor that is used to express the relationship between the currents.

In accordance with the domain decomposition paradigms, the MoM matrix equation $\mathbf{Z} \mathbf{J}=\mathbf{V}$ for the entire problem can be block-partitioned as

$$
\left[\begin{array}{cccc}
\mathbf{Z}_{11} & \mathbf{Z}_{12} & \cdots & \mathbf{Z}_{1 M} \\
\mathbf{Z}_{21} & \mathbf{Z}_{22} & \cdots & \mathbf{Z}_{2 M} \\
\vdots & \ddots & \cdots & \vdots \\
\mathbf{Z}_{M 1} & \cdots & \cdots & \mathbf{Z}_{M M}
\end{array}\right]\left[\begin{array}{c}
\mathbf{J}_{1} \\
\mathbf{J}_{2} \\
\vdots \\
\mathbf{J}_{M}
\end{array}\right]=\left[\begin{array}{c}
\mathbf{V}_{1} \\
\mathbf{V}_{2} \\
\vdots \\
\mathbf{V}_{M}
\end{array}\right] .
$$

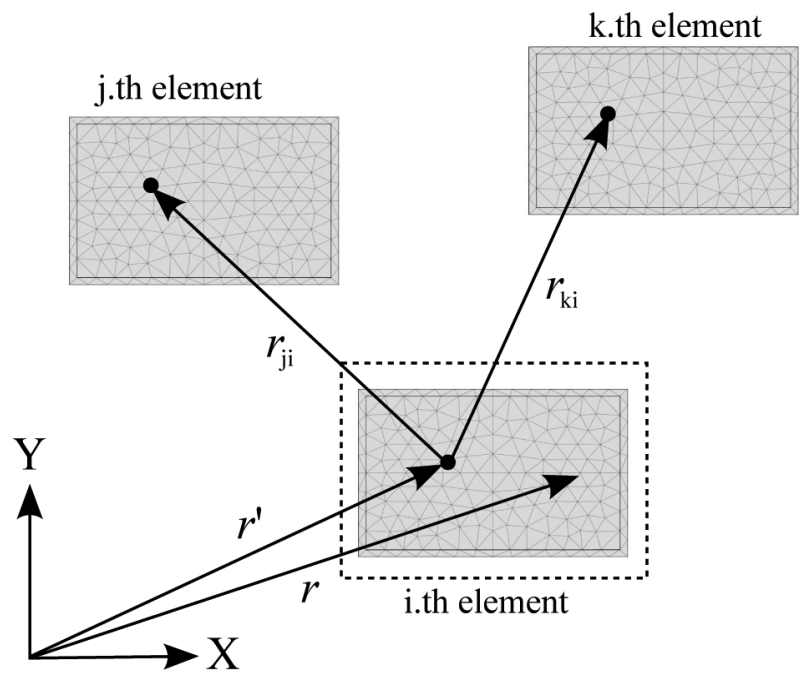

Fig. 1. Antenna array consisting of $M$ elements with $N_{i}$ unknowns each.

By using (1), we can calculate the total active current distribution, i.e., the solution to(2), by solving smaller block matrices. To illustrate this, consider that by applying(1), the currents on domains $2, \ldots, M$ may be expressed in terms of the current on domain 1 as

$$
\mathbf{J}_{2}=\alpha_{21} \mathbf{J}_{1} ; \quad \mathbf{J}_{3}=\alpha_{31} \mathbf{J}_{1} ; \quad \mathbf{J}_{M}=\alpha_{M 1} \mathbf{J}_{1} .
$$

By substituting (3) in (2), we can then solve for the active current distribution on domain 1 , i.e.,

$$
\begin{aligned}
\mathbf{V}_{1} & =\left[\mathbf{Z}_{11}+\left(\alpha_{21} \mathbf{Z}_{12}+\cdots+\alpha_{M 1} \mathbf{Z}_{1 M}\right)\right] \mathbf{J}_{1} \\
& =\left[\sum_{m=1}^{M} \alpha_{m 1} \mathbf{Z}_{1 m}\right] \mathbf{J}_{1}=\mathbf{Z}_{1}^{\text {act }} \mathbf{J}_{1}
\end{aligned}
$$

where the excitation-dependent $\mathbf{Z}_{1}^{\text {act }}$ is called the active impedance matrix, that accounts for both the self-coupling as well as the mutual coupling from the surrounding active array environment.

On a similar basis, we can calculate the active impedance matrices for each of the array elements. In general, the active impedance matrices of the domains are different, i.e., $\mathbf{Z}_{n}^{\text {act }} \neq \mathbf{Z}_{m}^{\text {act }}$ for $n \neq m$. The resulting current distribution on the array elements $n$ and $m$ is therefore perturbed in the sense that $\mathbf{J}_{n} \neq \alpha_{n m} \mathbf{J}_{m}$ for $n \neq m$ as per our initial assumption.

The runtime for the DGFM scales as $\mathcal{O}\left(M \times N_{i}^{3}\right)$ as opposed to $\mathcal{O}\left(\left(M \times N_{i}\right)^{3}\right)$ should the MoM be used for the entire structure. Likewise, the memory usage scales according to $\mathcal{O}\left(N_{i}^{2}\right)$ and not $\mathcal{O}\left(\left(M \times N_{i}\right)^{2}\right)$. It should be noted that the DGFM technique presented here does not allow for the calculation of embedded element patterns (EEP), as it would mean that all neighboring elements are not excited, i.e., $C_{k}=0$ in (1). Hence, and in contrast to an all-excited array, the effect of mutual coupling due to a local excitation is not accounted for. The same limitation applies to calculating the scattering matrix for the array. Appendix A discusses a method for correcting the excitation coefficients, $C_{k}$, so that first-order coupling effects are also accounted for whenever spatially localized solutions for the current are to be expected. The aforementioned ensures that $C_{k} \neq 0$ 
even for the case where only one element is excited. In the following, however, we will focus on the all-excited array case.

\section{B. Comparing the DGFM With MBF Techniques}

The DGFM shares a few commonalities with macro basis function (MBF) techniques such as the characteristic basis function method (CBFM) [14], [17] that are based on partitioning the problem into a number of smaller subdomains. A comparison between the DGFM and the CBFM, as presented in [3] for disjoint domains, will be discussed here.

For the CBFM, the total unknown current distribution on a domain can be expressed as a linear combination of primary and secondary $\mathrm{CBFs},{ }^{2}$ each weighted with an unknown complex constant, $\beta_{n}$ [3]. For illustration, consider the current on domain 1 , which may then be expressed as

$$
\mathbf{J}_{1} \simeq \beta_{11} \underbrace{\mathbf{J}_{11}}_{\text {Prim.CBF }}-\sum_{m=2}^{M} \beta_{1 m} \underbrace{\mathbf{Z}_{11}^{-1} \mathbf{Z}_{1 m} \mathbf{J}_{1 m}}_{\text {Sec.CBFs }} .
$$

The first term in (5) is the primary $\mathrm{CBF}$ and is calculated as the current on the subdomain in isolation and the secondary CBFs, i.e., the terms $m=2, \ldots, M$, represents the current induced on subdomain 1 , by using as excitation the primary CBFs of the surrounding elements.

For the DGFM, the initial current distributions that are assumed on each of the domains, are taken as the primary CBFs only. Note, however, that these primary CBFs are not generated explicitly beforehand. For the DGFM, we require only the relative relationship between the current on domains $p$ and $q$, i.e., $\mathbf{J}_{p}$ and $\mathbf{J}_{q}$ which may be expressed as ( $c f$. Appendix A)

$$
\alpha_{p q} \simeq \frac{\left\langle\Lambda, \mathbf{J}_{p 1}\right\rangle}{\left\langle\Lambda, \mathbf{J}_{q 1}\right\rangle}
$$

for $p, q=1,2 \ldots, M$, where $\mathbf{J}_{p 1}$ and $\mathbf{J}_{q 1}$ are the primary CBFs on domains $p$ and $q$, respectively, and $\boldsymbol{\Lambda}$ is a testing vector (e.g., a vector filled by ones). As illustrated in (6), the ratio between the primary CBFs is in turn identical to the ratio of the applied voltage excitation coefficients of the subdomains $p$ and $q$, i.e., the $\alpha$-coefficients defined in (1). The DGFM therefore does not require the initial calculation of a set of primary $\mathrm{CBFs}$, which is of $\mathcal{O}\left(M \times N_{i}^{3}\right)$ and increases to $\mathcal{O}\left(M \times N_{i}^{3}+\left(M \times N_{i}\right)^{2}\right)$ when adding also secondary CBFs, as discussed in [19], at the cost of compromising the solution accuracy only slightly.

The next difference between the CBFM and the DGFM is clear when comparing the $M^{2} \times M^{2}$ reduced impedance matrix setup for the CBFM [3], which represents the interaction between the CBFs generated on each of the $M$ domains, with the active impedance matrix setup for the DGMF as explained in Section II-A. Following [17], the CBFM reduced matrix entries may be calculated as

$$
\mathbf{Z}_{p q}^{\mathrm{CBF}}=\left\langle\mathbf{J}_{p m}^{T}, \mathbf{Z}_{p q}^{\mathrm{RWG}} \mathbf{J}_{q n}\right\rangle
$$

with $\mathbf{Z}_{p q}^{\mathrm{RWG}}$ the coupling submatrix also used in (2).

Accurate coupling is modeled with (7), by accounting for the interaction between two CBFs using the full-wave coupling

\footnotetext{
${ }^{2}$ In [18] tertiary CBFs were also used to model a connected patch array.
}

matrix, $\mathbf{Z}_{p q}^{\mathrm{RWG}}$. For the DGFM, mutual coupling between the domains is approximated by weighting $\mathbf{Z}_{p q}^{\mathrm{RWG}}$ with the known $\alpha_{p q}$-coefficient. In terms of accuracy, the DGFM does therefore not account for spatially localized first-order coupling effects, as in the CBFM; however, it still accounts for spatially extended global coupling effects on account of the infinite array assumption, unlike the CBFM. Another difference that can be noted, is that the CBFM allows for better treatment of multiple excitations (MRHS), since the active impedance matrices in (4) have to be recalculated for every array excitation.

The benefit to using the DGFM is clear when comparing the computational costs, in terms of runtime and memory usage, with that of the CBFM. As already mentioned, the DGFM does not require the initial calculation of a set of primary and secondary CBFs. Another difference is the cost associated with calculating the active impedance matrices compared to that of setting up the reduced matrix equation. For the CBFM, calculating (7) for each of the domains $p=1, \ldots, M$ and $q=$ $1, \ldots, M$ scales as $\mathcal{O}\left(M^{4} \times N_{i}^{2}\right)$. The cost of calculating the active impedance matrix equations in (4), scales as $\mathcal{O}\left(M^{2} \times N_{i}^{2}\right)$, i.e., a saving of $\mathcal{O}\left(M^{2}\right)$.

It is however to be noted that the effect of applying techniques such as the ACA [17], that accelerates the matrix vector product in (7), by approximating $\mathbf{Z}_{p q}^{\mathrm{RWG}}$ as a low-rank decomposition, $\tilde{\mathbf{Z}}_{p q}^{\mathrm{RWG}}$, has not been considered. In [20], the cost of calculating the reduced impedance matrix has also been addressed by using a low-order harmonic-polynomial function as a compact representation for the reduced matrix entries in (7). This harmonic polynomial expression is obtained by precomputing the reaction integrals in a limited set of relative positions between domains.

In addition, $M$ CBFs per domain are typically not used, as the number of CBFs per domain can be reduced by applying a Gram-Schmidt orthogonalization [3], or a singular value decomposition (SVD) [17] to retain only a limited number of orthonormal CBFs. If we consider on average $L$ CBFs per domain, with $L<M$, then the cost for calculating the reduced impedance matrix in (7) reduces from $\mathcal{O}\left(M^{4} \times N_{i}^{2}\right)$ to $\mathcal{O}\left(M^{2} \times\right.$ $L^{2} \times N_{i}^{2}$ ). It should however be noted that the cost of applying the Gram-Schmidt orthogonalization, or the SVD, to reduce the number of CBFs per domain adds additional complexity to the CBFM algorithm as explained in [19].

The CBFM and the DGFM also differ in terms of memory usage. The memory requirement for calculating the primary CBFs scales as $\mathcal{O}\left(N_{i}^{2}\right)$, as we need to store the LU factorization of the self-interaction matrix of each domain, i.e., $\left(\mathbf{Z}_{m m}^{\mathrm{RWG}}\right)^{-1}$ that is of size $N_{i} \times N_{i}$. Another $\mathcal{O}\left(N_{i}^{2}\right)$ is required for storing the coupling matrices, $\mathbf{Z}_{p q}^{\mathrm{RWG}}$, used when generating the secondary CBFs in (5) and also when calculating the reduced matrix entries in (7). In addition to the aforementioned, storing the CBFM reduced impedance matrix scales as $\mathcal{O}\left(M^{4}\right)$. The memory requirement for the DGFM scales only as $\mathcal{O}\left(N_{i}^{2}\right)$ for storing the active impedance matrix of each domain. The computational complexity of the DGFM and CBFM formulation, as presented in [3] for disjoint arrays, is summarized in Table I.

The computational complexities of the CBFM and DGFM algorithms have been qualitatively compared in this section, and their relative accuracies have been examined. It has been 
TABLE I

Order of CPU-Time And Memory Usage for Certain Phases of THE CBFM AND DGFM WHEN APPLIED TO THE ARRAY GEOMETRY of Fig. 1, Consisting of $M$ Disjoint Elements Each With $N_{i}$ SUBSECTIONAL BASIS FUnCTIONS

\begin{tabular}{|c|c|c|}
\hline Method: & CBFM & DGFM \\
\hline $\begin{array}{c}\text { MoM matrix } \\
\text { setup time }\end{array}$ & $\mathcal{O}\left(\left(M \times N_{i}\right)^{2}\right)$ & $\mathcal{O}\left(\left(M \times N_{i}\right)^{2}\right)$ \\
\hline $\begin{array}{c}\text { Calculation of } \\
\text { Primary basis }\end{array}$ & $\mathcal{O}\left(M \times N_{i}^{3}\right)$ & $N A$ \\
\hline $\begin{array}{c}\text { Calculation of } \\
\text { Secondary basis }\end{array}$ & $\mathcal{O}\left(\left(M \times N_{i}\right)^{2}\right)$ & $N A$ \\
\hline $\begin{array}{c}\text { Calculation of } \\
\text { reduced matrix }\end{array}$ & $\mathcal{O}\left(M^{4} \times N_{i}^{2}\right)$ & $N A$ \\
\hline $\begin{array}{c}\text { Calculation of active } \\
\text { impedance matrices }\end{array}$ & $N A$ & $\mathcal{O}\left(M^{2} \times N_{i}^{2}\right)$ \\
\hline $\begin{array}{c}\text { Memory usage } \\
\text { (whole solution) }\end{array}$ & $\mathcal{O}\left(2 N_{i}^{2}+M^{4}\right)$ & $\mathcal{O}\left(N_{i}^{2}\right)$ \\
\hline
\end{tabular}

shown that although the DGFM is less accurate than MBF techniques - such as the CBFM for cases where higher order coupling is required - one gains a saving in both memory usage and runtime for cases where it can be used.

Finally, it is worth noting that the DGFM can be enhanced by the CBFM for the analysis of large disjoint arrays consisting of subarray tiles, as explained in [21].

\section{AcCelerating the Active Impedance MATRIX CALCULATION}

As noted in Section II-B, calculating the MoM impedance matrix for the $M$ element array scales as $\mathcal{O}\left(\left(M \times N_{i}\right)^{2}\right)$, and can rapidly become the dominant part in the solution phase for the DGFM, especially for large arrays. In the following sections, we explore a scheme for reducing this cost, viz., by truncating the summation that is present in the calculation of the active impedance matrices. This requires that the active impedance matrix terms in (4) be sorted beforehand, and is explained in the following subsection.

\section{A. Sorting the Terms of $\mathbf{Z}_{i}^{\text {Act }}$}

The active impedance matrix of the $i$ th domain may be expressed as follows:

$$
\begin{aligned}
\mathbf{Z}_{i}^{\text {act }} & =\mathbf{Z}_{i i}+\alpha_{1 i} \mathbf{Z}_{i 1}+\alpha_{2 i} \mathbf{Z}_{i 2}+\cdots+\alpha_{M i} \mathbf{Z}_{i M} \\
& =\sum_{k=1}^{M} \alpha_{k i} \mathbf{Z}_{i k}
\end{aligned}
$$

with $\alpha_{m n}$ as defined in (1), i.e., $\alpha_{k i}=C_{k} / C i$, where $C_{k}$ and $C_{i}$ are the applied excitation coefficients on domains $k$ and $i$, respectively.

In order to sort the terms in (8), the following scalar quantity is defined for each element:

$$
\zeta^{i}=\frac{\left|\alpha_{k i}\right|}{\left|\boldsymbol{r}_{k i}\right|} .
$$

Equation (9) therefore accounts for both the relative excitation magnitude and physical separation between the elements $k$ and $i$, respectively. The distance between the elements $\boldsymbol{r}_{k i}$, illustrated in Fig. 1, assumes that the elements are not rotated arbitrarily with respect to each other. This sorting is done prior to the analysis of each element and, for optimal performance, the Quicksort algorithm is used [22].

\section{B. Truncating the $\mathbf{Z}_{i}^{\text {Act }}{ }^{-S u m m a t i o n}$}

If (8) is sorted according to (9), then this would represent a decreasing function and the number of terms used in the summation can be truncated to some value, $M_{c}$, that defines the radius of convergence for each of the elements, i.e., $M_{c}=M \times R_{c}$, with $R_{c}$ a factor between 0 and 1 . The active impedance matrix is then approximated as follows:

$$
\begin{aligned}
\mathbf{Z}_{i}^{\text {act }} & =\underbrace{\mathbf{Z}_{i i}+\alpha_{1 i} \mathbf{Z}_{i 1}+\alpha_{2 i} \mathbf{Z}_{i 2}}_{M_{c} \text { terms }}+\cdots+\alpha_{M i} \mathbf{Z}_{i M} \\
& \simeq \sum_{k=1}^{M_{c}} \alpha_{k i} \mathbf{Z}_{i k} .
\end{aligned}
$$

The complexity of the matrix-fill phase can therefore be reduced to $\mathcal{O}\left(\left(R_{c} \times M \times N_{i}\right)^{2}+M \times S\right)$, where $R_{c}$ is the convergence radius between 0 and 1 , and $S$ is a small factor related to the sorting of the DGF terms, using (9).

Numerical results will be illustrated in Section V, which will discuss the effect of truncating the active impedance matrix summation.

\section{Parallelization OF THE DGFM Using HYBRID MPI/OPENMP}

The DGFM is particularly suited to a distributed parallelization strategy using routines such as the message passing interface (MPI) [23]. Each parallel process that forms part of the computational domain is allocated a certain number of array elements to analyze. The final result, i.e., the active current distribution for the entire array, is then combined at the end, when each process has completed the analysis of its subset of the problem.

Inherent to such a distributed programming model, is the fact that each process is allocated its own section of memory, which in turn leads to unnecessary overhead in the event that the processes are located on the same machine, operating in a shared memory environment. Another parallel programming paradigm, OpenMP [24], has emerged in recent years, which allows one to run multiple threads of one process in parallel on such an architecture. What makes OpenMP attractive is the avoidance of memory duplication if multiple parallel processes/threads need to access the same data.

The DGFM uses a hybrid OpenMP/MPI parallelization scheme, whereby the MPI library is used for distributed parallelization and OpenMP threading for intra-node communication. The aforementioned is currently applied to the LU-decomposition of the active impedance matrices.

\section{NUMERICAL RESULTS}

To illustrate the efficiency of the simulation techniques described in the previous subsection, irregular-spaced sparse arrays of log-periodic type structures are analyzed. The element used is called the Zig-Zag, and is explained in more detail in Section V-A. 


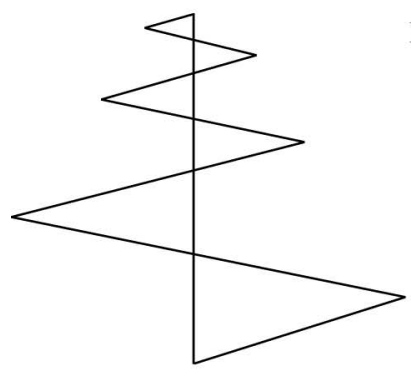

Feed

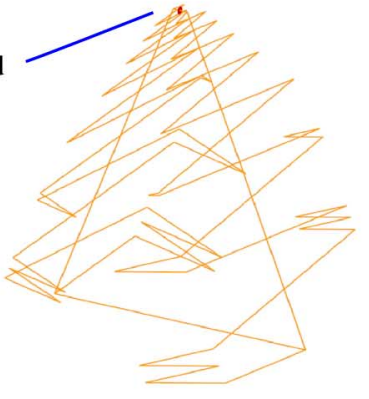

Single Polarization

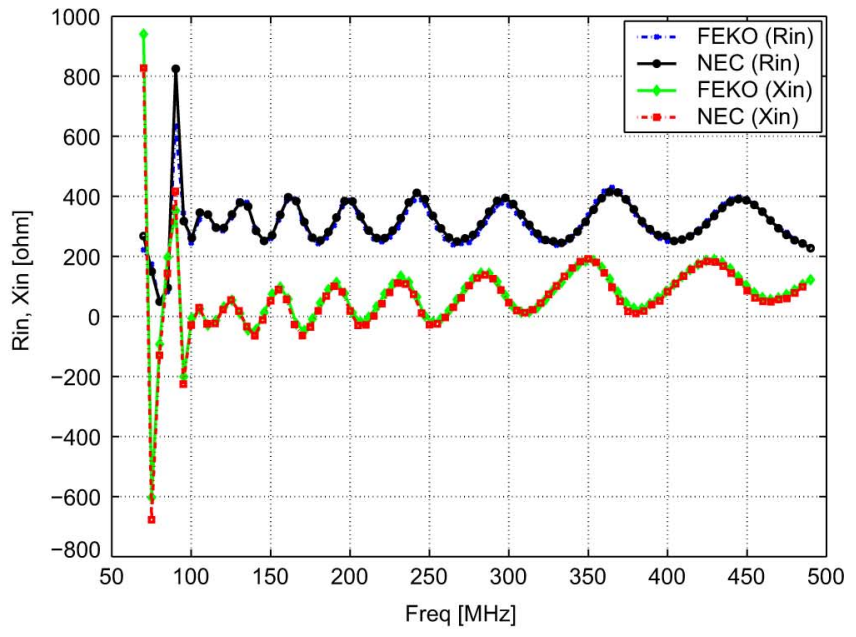

(b)

Fig. 2. (a) Schematics representation and single-polarization of the Zig-Zag antenna and (b) the real and imaginary part of the input impedance of the singlepolarized element simulated with FEKO and NEC, respectively. (a) Schematic representation of the Zig-Zag geometry and a single-polarized antenna with the arms folded. (b) Real and imaginary parts of the input impedance for the single-polarized Zig-Zag antenna, simulated with FEKO and NEC.

\section{A. Test Example 1: the Zig-Zag Array Element}

The element used in the following array configurations is called the Zig-Zag. The geometry is derived from that of a logperiodic antenna, as introduced by DuHamel [15]. Here, the geometry is simplified to a triangular tooth or Zig-Zag structure, as proposed by DuHamel in [25]. The Zig-Zag geometry is presented in Fig. 2(a) where the trapezoidal tooth structure is illustrated (left) as well as the manner in which it is folded to obtain a single-polarized antenna. The Zig-Zag element is folded to limit the footprint of the antenna to $1 \mathrm{~m}^{2}[26]$ in accordance with the SKA1-low specifications [1].

The real and imaginary components of the input impedance of the single-polarized (folded) Zig-Zag element are presented in Fig. 2(b). The results were obtained with FEKO [13] and the numerical electromagnetics code (NEC) [27], respectively.

The input impedance oscillates around $300 \Omega$ over a fairly wide frequency band, ranging from $70 \mathrm{MHz}$ to $450 \mathrm{MHz}$, in line with the requirements of the SKA1-low frequency range. Two single-polarized elements can also be rotated $90^{\circ}$ with respect to each other, in order to achieve dual-polarization. In the next section, an array of such dual-polarized Zig-Zag elements will be analyzed with the DGFM.

\section{B. Test Example 2: A 26-Element Array of Dual-Polarized Zig-Zag Antennas}

To illustrate the effect of limiting the mutual coupling during the calculation of $\mathbf{Z}_{1}^{\text {act }}$ to the $N_{c}$ dominating elements, a 26 element array consisting of dual-polarized Zig-Zag antennas, illustrated in Fig. 3(a), is analyzed using the MoM and DGFM with $R_{c}$ values of $100 \%, 50 \%$ and $0 \%$, respectively. For $R_{c}=$ $0 \%$, only self-coupling for the element is assumed. The element spacing ranges from between $\lambda / 2$ to $3 \lambda$ at $70 \mathrm{MHz}$. Although spacings of $\lambda / 2$ are clearly denser than normally needed in an irregularly spaced array, it is chosen as such to illustrate the effect of various $R_{c}$ values on the accuracy of the DGFM. The element is discretized by using 325 elementary basis functions, leading to a total of 8450 unknowns.
The far-field gain pattern is analyzed along the ZX-plane for scan-angles of $\theta=0^{\circ}$ and $\theta=60^{\circ}$, and is illustrated in Fig. 3(c) and (d). The DGFM results for $R_{c}=100 \%$ compare well with those obtained by the MoM solution. Included in the results are those obtained for $R_{c}=0 \%$ and $R_{c}=50 \%$ values. Fig. 3(e) and (f) illustrates the errors in $\mathrm{dB}$ for $R_{c}=0 \%$ and $R_{c}=50 \%$ compared to the DGFM with $R_{c}=100 \%$. As can be noted in Fig. 3(e) and (f), the error is slightly larger than $1 \mathrm{~dB}$ for certain off-broadside angles. The reason for the aforementioned, is as follows. For a slowly varying excitation law, the initial condition that the currents be identical on all subdomains, except for a complex scaling factor, is particularly true for near-broadside scan angles and for antenna elements that support only a low number of antenna modal currents. These limitations were also observed in [28], where a similar perturbation approach has been described for the CBFM.

The computational complexity of the DGFM compared to the MoM is summarized in Table II. The matrix fill-time for the conventional DGFM (i.e., with $R_{c}=100 \%$ ) is similar to that of the MoM; however significant savings can be observed in the matrix solution phase (which is dominated by the LU-decomposition) as well as in the memory usage.

Table III summarizes the runtimes for the matrix fill phase, associated with the various $R_{c}$ values. From the results it can be concluded that the accuracy of the DGFM can be controlled by changing the value of $R_{c}$.

To illustrate the efficiency of the parallelization of the DGFM as discussed in Section IV, the 26-element array was simulated using five compute nodes. The measured runtime speedup is shown in Fig. 4 and compares well to the ideal case.

\section{Test Example 3: A 529-Element Irregularly Spaced Array of Zig-Zag Elements}

Following the methodologies explained in Sections III and IV, a larger 529-element dual-polarized Zig-Zag array was analyzed by using eight distributed parallel processes on a Linux 


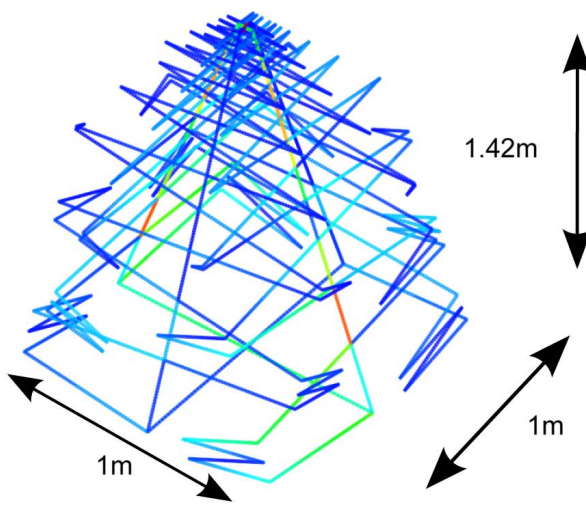

(a)

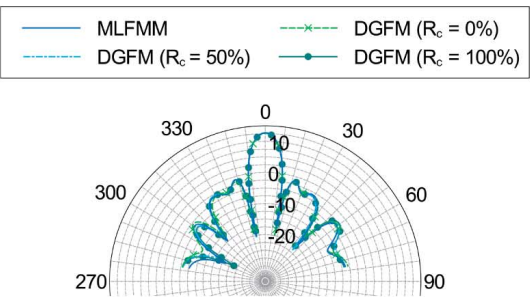

(c)

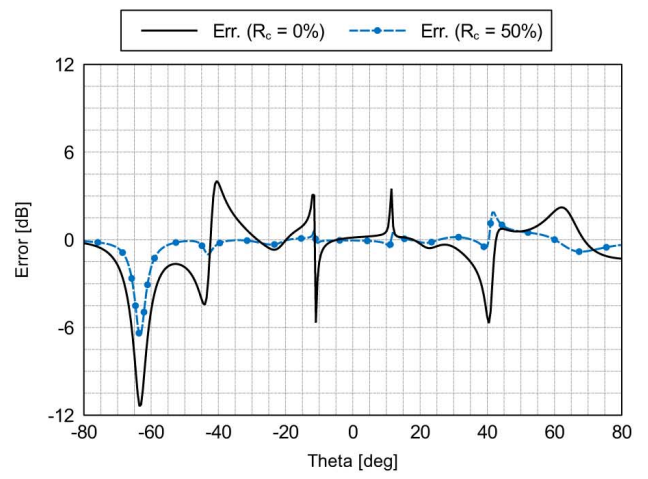

(e)

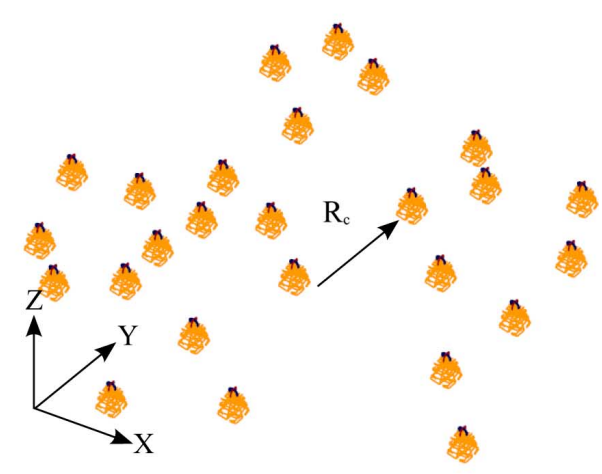

(b)

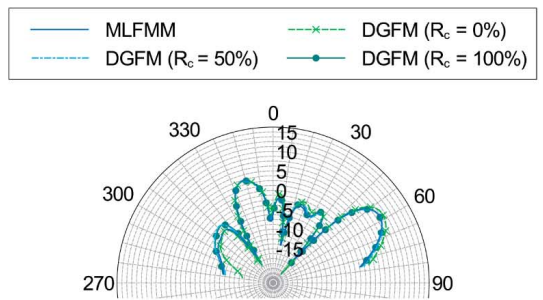

(d)

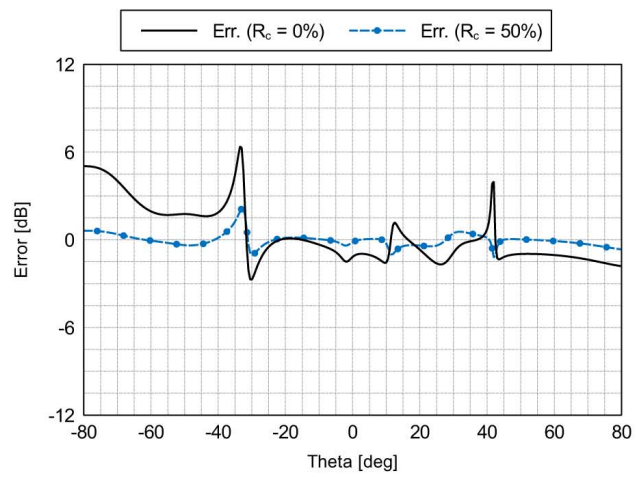

(f)

Fig. 3. Application of the DGFM to a Zig-Zag antenna displayed in (a), in an array configuration displayed in (b). The directivity patterns for scan-angles of $\left(\theta=0^{\circ}, \phi=0^{\circ}\right)$ and $\left(\theta=60^{\circ}, \phi=0^{\circ}\right)$ are presented in (c) and (d), respectively. The errors in the directivity for different $R_{c}$ values are presented in (e) and (f), where $R_{c}=100 \%$ is used as reference. All results are obtained for the active array environment where all the elements are excited equally and simultaneously. (a) Dual-polarized Zig-Zag element geometry. (b) Array configuration containing 26 irregularly spaced Zig-Zag elements. The element spacings ranges from $\lambda / 2$ to $3 \lambda$, at an operating frequency of $70 \mathrm{MHz}$. (c) Total directivity pattern (dBi) for a scan-angle of $\theta=0^{\circ}, \phi=0^{\circ}$. (d) Total directivity pattern (dBi) for a scan-angle of $\theta=60^{\circ}, \phi=0^{\circ}$. (e) Error (dB) in the calculated directivity for $R_{c}=0 \%$ and $R_{c}=50 \%$ compared to $R_{c}=100 \%$. Considered scan angle: $\left(\theta=0^{\circ}, \phi=0^{\circ}\right)$. (f) Error (dB) in calculated directivity for $R_{c}=0 \%$ and $R_{c}=50 \%$ compared to $R_{c}=100 \%$. Considered scan angle: $\left(\theta=60^{\circ}, \phi=0^{\circ}\right)$.

TABLE II

RunTime AND MEMORY REQUiREMENT FOR SimULATING A 26-ELEMENT ZIG-ZAG ARRAY USING THE MOM AND DGFM

\begin{tabular}{|c|c|c|}
\hline Method: & MoM & $\begin{array}{c}\text { DGFM } \\
\left(R_{c}=100 \%\right)\end{array}$ \\
\hline $\begin{array}{c}\text { Time for } \\
\text { matrix setup }\end{array}$ & $326.24 \mathrm{sec}$ & $379.69 \mathrm{sec}$ \\
\hline $\begin{array}{c}\text { Time for } \\
\text { matrix solution }\end{array}$ & $188.616 \mathrm{sec}$ & $0.83 \mathrm{sec}$ \\
\hline $\begin{array}{c}\text { Memory usage } \\
\text { (whole solution) }\end{array}$ & $1.072 \mathrm{GByte}$ & $7.56 \mathrm{MByte}$ \\
\hline
\end{tabular}

EM64T platform with $2 \mathrm{x}$ quad core processors. The array geometry is illustrated in Fig. 5. The array consists of 171925 elementary basis functions with inter-element spacings again ranging from between $\lambda / 2$ and $3 \lambda$, at an operating frequency of $70 \mathrm{MHz}$. Convergence threshold values of $R_{c}=0 \%$ and $R_{c}=$ $50 \%$ were used for the analysis. The computational complexity of the simulations is summarized in Table IV. The far-field gain

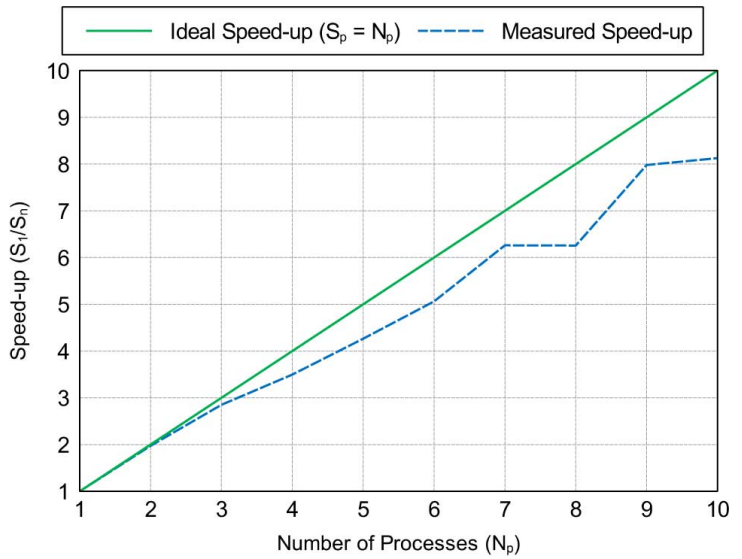

Fig. 4. Runtime speedup measured for the 26-element Zig-Zag element array.

patterns for $\theta=0^{\circ}$ and $\theta=60^{\circ}$ are illustrated in Fig. 5. From the results it can be seen that the errors in the modeled 


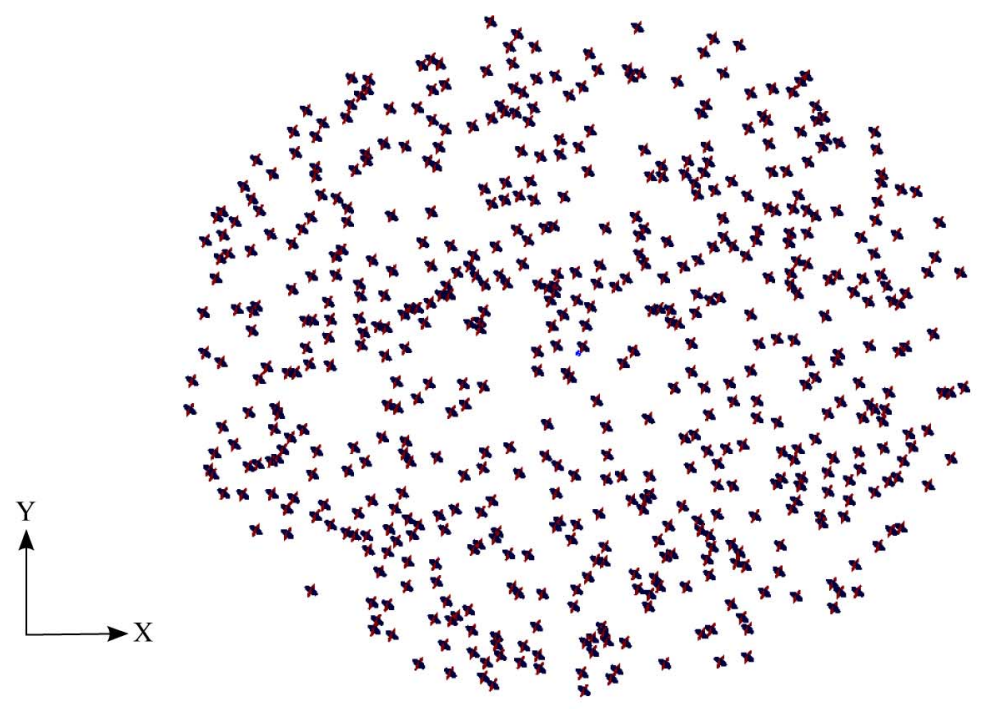

(a)

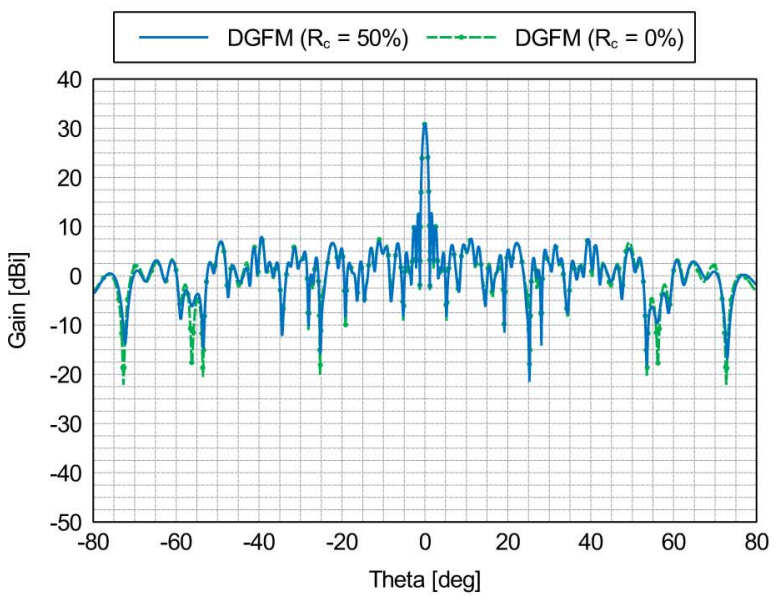

(b)

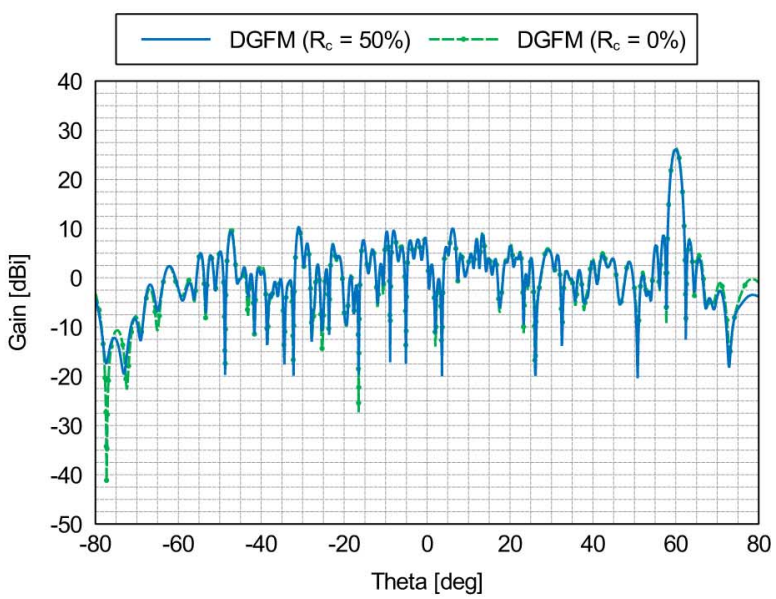

(c)

Fig. 5. Applying the DGFM to the Zig-Zag antenna displayed in Fig. 3(a) in a 529-element array configuration. The gain patterns for scan-angles of $\left(\theta=0^{\circ}\right.$, $\left.\phi=0^{\circ}\right)$ and $\left(\theta=60^{\circ}, \phi=0^{\circ}\right)$ are presented in (b) and (c), respectively. All results are obtained for the active array environment where all the elements are excited equally and simultaneously. (a) Array configuration containing 529 irregularly spaced Zig-Zag elements. The element spacings ranges from $\lambda / 2$ to $3 \lambda$, at an operating frequency of $70 \mathrm{MHz}$. (b) Total gain pattern (dB) for a scan-angle of $\theta=0^{\circ}, \phi=0^{\circ}$. (c) Total gain pattern (dB) for a scan-angle of $\theta=60^{\circ}$, $\phi=0^{\circ}$.

TABLE III

Runtime AND MEMORY ReQUiREMENT FOR SIMULATING A 26-ELEMENT Zig-ZAG ARRAY Using DifFERENT $R_{c}$ VALUES

\begin{tabular}{|c|c|c|c|}
\hline Method: & $\begin{array}{c}\text { DGFM } \\
\left(R_{c}=100 \%\right)\end{array}$ & $\begin{array}{c}\text { DGFM } \\
\left(R_{c}=50 \%\right)\end{array}$ & $\begin{array}{c}\text { DGFM } \\
\left(R_{c}=0 \%\right)\end{array}$ \\
\hline $\begin{array}{c}\text { Number of coupling } \\
\text { terms for DGF }\left(N_{c}\right)\end{array}$ & 29 & 15 & 1 \\
\hline $\begin{array}{c}\text { Time for } \\
\text { matrix setup }\end{array}$ & $379.69 \mathrm{sec}$ & $199.43 \mathrm{sec}$ & $1.22 \mathrm{sec}$ \\
\hline
\end{tabular}

mutual coupling only have a minor effect for $-80^{\circ} \leq \theta \leq$ $-50^{\circ}$ and $50^{\circ} \leq \theta \leq 80^{\circ}$, and even less for $\pm 45^{\circ}$ off-vertical for SKA1-low. The technique introduced in this paper has permitted a computationally efficient investigation to be undertaken. This can be applied to other candidate antennas, such as the log periodic proposed in [16].

\section{CONCLUSION AND RECOMMENDATIONS}

In this work, the DGFM was presented as a technique that may be applied to large irregular array configurations, such as
TABLE IV

Runtimes AND MEMORY ReQUiREMENTS FOR THE ANALYSIS OF THE ZIG-ZaG ARRAY CONFIGURATION OF Fig. 5 ON A LinuX EM64T PlatForm With 2 X Intel Xeon Quad Core Processors at 2.67-GHz Clock RATE EACH. THE DGFM WITH $R_{c}=0 \%$ AND $R_{c}=50 \%$ WAS USED, With Eight Parallel Processes

\begin{tabular}{|c|c|c|}
\hline Method: & $\begin{array}{c}\text { DGFM } \\
\left(R_{c}=0 \%\right)\end{array}$ & $\begin{array}{c}\text { DGFM } \\
\left(R_{c}=50 \%\right)\end{array}$ \\
\hline $\begin{array}{c}\text { Total solution } \\
\text { time }\end{array}$ & $14.23 \mathrm{~min}$ & 2.27 hours \\
\hline $\begin{array}{c}\text { Memory usage } \\
\text { (per process) }\end{array}$ & $143.1 \mathrm{MByte}$ & $143.1 \mathrm{MByte}$ \\
\hline
\end{tabular}

those being considered for the SKA low-frequency aperture arrays. The formulation of the DGFM is such that mutual coupling can be controlled by setting a convergence radius, $R_{c}$, for the elements being analyzed, as explained in Section III-A. In doing so, the matrix fill phase of the DGFM can be accelerated quite significantly. Future work would be directed towards obtaining an optimal value for $R_{c}$, based on the array geometry, as well as including the support for arbitrary rotations of the array 
elements. In Section IV, it was also indicated how the DGFM can be accelerated by using parallel programming. At the time of writing, only the matrix solution phase of the DGFM, i.e., the LU-decomposition, supported parallelization with OpenMP threading. A further extension to this part of the DGFM, would be also to apply this shared memory programming paradigm to the matrix-fill phase, which was discussed in Section III-A.

In Appendix A, a method for improving the solution accuracy of the DGFM has been discussed to more accurately incorporate spatially concentrated coupling effects in addition to the global coupling solutions (spectral Floquet waves) that are inherent to the infinite-array-type of assumption in the DGFM. The improvement is based on the Jacobi decomposition of the block factorized MoM matrix which not only allows for the analysis of more concentrated localized excitation schemes - or more rapidly varying currents on the array elements - but also the calculation of embedded element patterns (EEPs) and reveals commonalities with other MBF methods such as the CBFM. Since the focus in this paper is on the all-excited sparse array case of weakly coupled antenna elements, the aforementioned will be investigated more rigorously in future work.

\section{APPENDIX \\ DGFM IN RELATION TO JACOBI-GENERATED CBFS}

A connection is shown between the effective domain excitation coefficients $C_{k}$ in the DGFM and the multiple-scattering $\mathrm{CBF}$ in the CBFM as well as a manner in which the accuracy of the DGFM can be improved. The ideas are based on the Jacobi iterative solver as described in [29], [30], while the connection between Krylov subspace solvers and macro-basis functions have been described more recently in [31]. We begin by writing the block-partitioned matrix (2) as $\left(\mathbf{Z}^{\text {on }}+\mathbf{Z}^{\text {off }}\right) \mathbf{J}=\mathbf{V}$, where $\mathbf{Z}^{\text {on }}$ is the block-diagonal part of $\mathbf{Z}$, while $\mathbf{Z}^{\text {off }}$ contains the off-diagonal moment matrix blocks. Upon multiplying both sides by $\left(\mathbf{Z}^{\text {on }}\right)^{-1}$, and by realizing that $\mathbf{J}_{0}=\left(\mathbf{Z}^{\text {on }}\right)^{-1} \mathbf{V}$ is the induced current on the array structure in the absence of subdomain coupling, one obtains

$$
\begin{aligned}
\mathbf{J} & =\left[\mathbf{I}+\left(\mathbf{Z}^{\text {on }}\right)^{-1} \mathbf{Z}^{\text {off }}\right]^{-1} \mathbf{J}_{0} \\
& =\sum_{n=0}^{\infty}\left[-\left(\mathbf{Z}^{\text {on }}\right)^{-1} \mathbf{Z}^{\text {off }}\right]^{n} \mathbf{J}_{0}
\end{aligned}
$$

where the term $\left[\mathbf{I}+\left(\mathbf{Z}^{\text {on }}\right)^{-1} \mathbf{Z}^{\text {off }}\right]^{-1}$ has been rewritten with the aid of the infinite geometric series $(1-r)^{-1}=\sum_{n=0}^{\infty} r^{n}$. Convergence is achieved if the magnitude of the eigenvalue of the principal eigenvector of $\left(\mathbf{Z}^{\text {on }}\right)^{-1} \mathbf{Z}^{\text {off }}$ is less than unity [12], which is typically the case for radiating disjoint subdomains as considered in this work, for which the successively induced currents are expected to decay rapidly in an iterative multi-scattering context. By using the block partitioning in (2), the fullwave MoM solution (11) can also be written as

$$
\left[\begin{array}{c}
\mathbf{J}_{1} \\
\mathbf{J}_{2} \\
\vdots \\
\mathbf{J}_{M}
\end{array}\right]=\left[\begin{array}{c}
\mathbf{J}_{01}-\sum_{m=1, m \neq 1}^{M} \mathbf{z}_{11}^{-1} \mathbf{Z}_{1 m} \mathbf{J}_{0 m}+\cdots \\
\mathbf{J}_{02}-\sum_{m=1, m \neq 2}^{M} \mathbf{z}_{22}^{-1} \mathbf{Z}_{2 m} \mathbf{J}_{0 m}+\cdots \\
\vdots \\
\mathbf{J}_{0 M}-\sum_{m=1, m \neq M}^{M} \mathbf{z}_{M M}^{-1} \mathbf{Z}_{M m} \mathbf{J}_{0 m}+\cdots
\end{array}\right]
$$

where $\mathbf{J}_{0 m}=\left(\mathbf{Z}_{m m}\right)^{-1} \mathbf{V}_{m}$ is the induced current on the $m$ th subdomain in isolation. Note that $\mathbf{J}_{0 m}$ corresponds to the primary CBF as generated by the CBFM for subdomain $m$. Furthermore, $\sum_{m=1, m \neq p}^{M} \mathbf{Z}_{p p}^{-1} \mathbf{Z}_{p m} \mathbf{J}_{0 m}$ is the contribution of all the secondary CBFs to the domain $p$ [see (5)]. Hence, the final MoM solution for the current on each subdomain is the sum of the primary, secondary, tertiary CBFs, and so on, with known excitation coefficients in accordance with the known voltage excitation vector $\mathbf{V}$ and the Jacobi-iterative method in (12).

We can now express the ratio of first-order accurate currents on the domains $p$ and $q$, by using the first two terms in the Jacobiiterative method in (12), as follows:

$$
\alpha_{p q} \simeq \frac{\left\langle\boldsymbol{\Lambda}, \mathbf{J}_{p}\right\rangle}{\left\langle\boldsymbol{\Lambda}, \mathbf{J}_{q}\right\rangle}=\frac{\left\langle\boldsymbol{\Lambda}, \mathbf{J}_{0 p}-\sum_{m=1, m \neq p}^{M} \mathbf{Z}_{p p}^{-1} \mathbf{Z}_{p m} \mathbf{J}_{0 m}\right\rangle}{\left\langle\boldsymbol{\Lambda}, \mathbf{J}_{0 q}-\sum_{m=1, m \neq q}^{M} \mathbf{Z}_{q q}^{-1} \mathbf{Z}_{q m} \mathbf{J}_{0 m}\right\rangle}
$$

for $p, q=1,2 \ldots, M$. As explained in Section II-B, ignoring the first-order coupling terms on the RHS of (13), results in the zeroth-order approximation $\alpha_{p q} \approx\left\langle\boldsymbol{\Lambda}, \mathbf{J}_{0 p}\right\rangle /\left\langle\boldsymbol{\Lambda}, \mathbf{J}_{0 q}\right\rangle$, (where $\boldsymbol{\Lambda}$ is a vector filled by ones). This is equivalent to taking the ratio of the initially induced currents (i.e., primary $\mathrm{CBFs}$ ) without accounting for mutual coupling effects. This is the same as taking the ratio of the applied voltage excitation coefficients of the subdomains $p$ and $q$, i.e., the initial assumption made for the DGFM in (1).

Equation (13) provides a means to compute the $\alpha_{p q}$ coefficients in (1) more accurately by including first-order coupling effects used to model spatially concentrated solutions more accurately in addition to the spatially extended solutions already incorporated in the infinite-array-type of assumption used by the DGFM. In the above, two relatively inexpensive Jacobi iterations need to be performed-possibly even limiting the inclusion of only the near-coupling effects-which is then followed by the DGFM for solving the system rapidly through an infinite-array-type assumption leading to a block-diagonal system of linear equations, as opposed to taking a large number of Jacobi iterations or building and solving a reduced matrix equation as in the CBFM. Furthermore, since the paper focusses on sparse arrays of all-excited weakly coupled antenna elements, the proposed refinement in (13) was unnecessary to achieve the results with an accuracy as reported in this paper. Finally, it is conjectured that (13) also allows for the introduction of an $\alpha$ scaling vector, rather than an $\alpha$ scaling factor used in (3), to increase the accuracy even further in case currents are expected to varying strongly between neighboring domains.

\section{ACKNOWLEDGMENT}

The authors would like to thank D. Schoemann for use of the Zig-Zag antenna models published in [26].

\section{REFERENCES}

[1] P. E. Dewdey, R. Millenaar, R. McCool, J. Lazio, and T. J. Cornwell, SKA1 System Baseline Design, SKA Office, Tech. Rep 2013.

[2] A. Ishimaru, R. J. Coe, G. E. Miller, and W. P. Geren, "Finite periodic structures approach to large scanning array problems," IEEE Trans. Antennas Propag., vol. 33, no. 11, pp. 95-100, Nov. 1985.

[3] V. V. Prakash and R. Mittra, "Characteristic basis function method: A new technique for efficient solution of method of moments matrix equations," Microw. Opt. Technol. Lett., vol. 36, pp. 95-100, 2003. 
[4] L. Matekovits, V. Laza, and G. Vecchi, "Analysis of large complex structures with the synthetic-functions approach," IEEE Trans. Antennas Propag., vol. 55, no. 9, pp. 2509-2521, Sep. 2007.

[5] D. Gonzalez-Ovejero, E. Acedo, N. Razavi-Ghods, and C. Craeye, "Fast MBF based method for large random array characterization," in IEEE Int. Symp. Antennas Propag. (APSURSI), 2009, pp. 1-4.

[6] F. Ling, C.-F. Wang, and J.-M. Jin, "An efficient algorithm for analyzing large scale microstrip structures using adaptive integral method combined with discrete complex-image method," IEEE Trans. Microw. Theory Technol., vol. 48, no. 11, pp. 832-839, Nov. 2000.

[7] T. Yuan, L. W. Li, and M. S. Leong, "Efficient analysis and design of finite phased arrays of printed dipoles using fast algorithm: Some case studies," J. Electromagn. Waves Applicat., vol. 21, no. 6, pp. 737-754, 2007.

[8] D. Ludick, U. Jakobus, and D. B. Davidson, "Efficient analysis of finite antenna arrays using the domain green's function method," in Proc. IEEE Int. Symp. Antennas Propag. (APSURSI), Jul. 2012, pp. $1-2$.

[9] D. Ludick, U. Jakobus, and D. B. Davidson, "Numerical analysis of finite antenna arrays using the domain green's function method," in Proc. Int. Conf. Electromagn. Adv. Applicat. (ICEAA), Sep. 2012, pp. 216-219.

[10] A. K. Skriverik and J. R. Mosig, "Analysis of finite phase arrays of microstrip patches," IEEE Trans. Antennas Propag., vol. 41, no. 8, pp. 1105-1114, Aug. 1993.

[11] A. K. Skriverik and J. R. Mosig, "Analysis of printed array antennas," IEEE Trans. Antennas Propag., vol. 45, no. 9, pp. 1411-1418, Sep. 1997.

[12] Y. Brand, A. K. Skrivervik, and J. R. Mosig, "An iterative scheme solution for the analysis of printed arrays," Microw. Opt. Technol. Lett., vol. 16, no. 2, pp. 106-115, 1997.

[13] "FEKO Suite 6.2-Field Computations Involving Bodies of Arbitrary Shape," EM Software \& Systems-S.A. (Pty) Ltd, Stellenbosch, South Africa, 2012 [Online]. Available: http://www.feko.info

[14] R. Mittra and K. Du, "Characteristic basis function method for iterationfree solution of large method of moments problems," Progress Electromagn. Res. B, vol. 6, pp. 307-336, 2008.

[15] R. DuHamel and F. Ore, "Logarithmically periodic antenna designs," IRE Int. Conv. Rec., vol. 6, pp. 139-151, 1958.

[16] E. de Lera Acedo, "SKALA: A log-periodic antenna for the SKA," in 2012 Int. Conf. Electromagn. Adv. Applicat. (ICEAA), Sep. 2012, pp. 353-356.

[17] R. Maaskant, R. Mittra, and A. Tijhuis, "Fast analysis of large antenna arrays using the characteristic basis function method and the adaptive cross approximation algorithm," IEEE Trans. Antennas Propag., vol. 56, no. 11, pp. 3440-3451, Nov. 2008.

[18] S. G. Hay, J. D. O'Sullivan, and R. Mittra, "Connected patch array analysis using the characteristic basis function method," IEEE Trans. Antennas Propag., vol. 59, no. 6, pp. 1828-1837, Jun. 2011.

[19] K. Konno, Q. Chen, K. Sawaya, and T. Sezai, "Optimization of block size for CBFM in MOM," IEEE Trans. Antennas Propag., vol. 50, no. 10, pp. 4719-4724, Oct. 2012.

[20] D. Gonzalez-Ovejero and C. Craeye, "Interpolatory macro basis functions analysis of non-periodic arrays," IEEE Trans. Antennas Propag., vol. 59, no. 8, pp. 3117-3122, Aug. 2011.

[21] D. Ludick, R. Maaskant, R. Mittra, U. Jakobus, and D. Davidson, “Applying the CBFM-enhanced DGFM to the analysis of large finite antenna arrays," in Proc. Int. Conf. Electromagn. Adv. Applicat. (ICEAA), 2013.

[22] W. Press, S. Teukolsky, W. Vetterling, and B. Flannery, Numerical recipes: The art of scientific computing, 3rd ed. Cambridge, U.K.: Cambridge Univ. Press, 2007.

[23] Argonne National Laboratory, Mathematical and Computer Science Division, "The message passing interface (MPI) standard," 2010 [Online]. Available: http://www.mcs.anl.gov/research/projects/mpi/ index.htm

[24] “The OpenMP API Specification for parallel programming," 2012 [Online]. Available: www.openmp.org

[25] R. DuHamel and D. Isbell, "Broadband logarithmically periodic antenna structures," IRE Int. Conv. Rec., vol. 5, pp. 119-128, 1957, IEEE.

[26] D. H. Schoeman, "Full scale low-cost ultra wide band antenna for SKA low frequency array," M.S. thesis, Stellenbosch Univ., Stellenbosch, South Africa, 2013.

[27] C. Burke and A. Poggio, "Numerical electromagnetics code (NEC) Method of moments," 1981.
[28] R. Maaskant, R. Mittra, and A. G. Tijhuis, "Fast solution of multi-scale antenna problems for the Square Kilometre Array (SKA) radio telescope using the characteristic basis function method (CBFM)," Appl. Comput. Electromagn. Soc. (ACES) J., vol. 24, no. 2, pp. 174-188, 2009.

[29] Y. Brand, A. K. Skrivervik, J. R. Mosig, and F. E. Gardiol, "New iterative integral equation technique for multilayered printed array antennas," in Math. Meth. Electromagn. Theory, Kharkov, Ukraine, Jun. 1998, pp. 615-617.

[30] A. C. Polycarpou, "Evaluation of stationary block iterative techniques for the solution of finite arrays using the fe-bi method and domain decomposition," in Proc. Eur. Conf. Antennas Propag. (EuCAP), Nice, France, 2006, pp. 1-6.

[31] N. Ozdemir, D. G. Ovejero, and C. Craeye, "On the relationship between multiple-scattering macro basis functions and Krylov subspace iterative methods," IEEE Trans. Antennas Propag., vol. 61, no. 4, pp. 2088-2098, Apr. 2013.

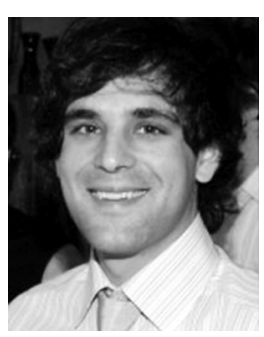

Daniel J. Ludick was born in the Free State, South Africa, on July 30, 1985. He received the B.Eng. degree in electrical and electronic engineering with computer science (cum laude) and the M.Sc.Eng. degree in electronic engineering (cum laude) from the University of Stellenbosch, Stellenbosch, South Africa, in 2007 and 2009, respectively. His master's thesis was focused on the efficient analysis of focal plane arrays for the Square Kilometre Array (SKA) radio telescope using electromagnetic simulation techniques. He is also currently pursuing the Ph.D. degree in electronic engineering at the University of Stellenbosch.

He is currently part of the development team for the computational electromagnetic software package, FEKO. His main research interests include computational electromagnetic simulation, domain decomposition techniques, and antenna design.

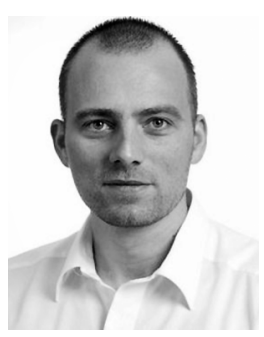

Rob Maaskant (M'11-SM'13) received the M.Sc. degree (cum laude) and the Ph.D. degree (cum laude), both in electrical engineering, from the Eindhoven University of Technology, Eindhoven, The Netherlands, in 2003 and 2010, respectively.

From 2003 to 2010, he was an Antenna Research Scientist at The Netherlands Institute for Radio Astronomy (ASTRON), Dwingeloo, The Netherlands, and from 2010 to 2012 as a Postdoctoral Researcher in the Antenna Group of the Signals and Systems Department at the Chalmers University of Technology, Gothenburg, Sweden, for which he won a Rubicon postdoctoral fellowship from the Netherlands Organization for Scientific Research (NWO), 2010. He is currently an Assistant Professor in the same Antenna Group. He is the primary author of the CAESAR software; an advanced integral-equation based solver for the analysis of large antenna array systems. His current research interest is in the field of receiving antennas for low-noise applications, meta-material based waveguides, and computational electromagnetics to solve these types of problems.

Dr. Maaskant received the second best paper prize ("best team contribution") at the 2008 ESA/ESTEC Workshop, Noordwijk, and was awarded a Young Researcher grant from the Swedish Research Council (VR), in 2011. He is an Associate Editor of the IEEE TRANSACTIONS ON ANTENNAS AND PROPAGATION. His Ph.D. dissertation was awarded "the best dissertation of the Electrical Engineering Department, 2010.”

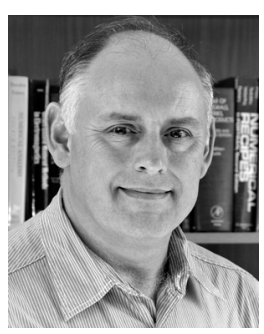

David B. Davidson (M'85-S'07-F'12) received the B.Eng, B.Eng (Hons), and M.Eng degrees (all cum laude) from the University of Pretoria, Pretoria, South Africa, in 1982, 1983, and 1986 respectively, and the Ph.D. degree from the University of Stellenbosch, Stellenbosch, South Africa, in 1991.

In 1988, he joined the University of Stellenbosch. As of 2011, he holds the South African Research Chair in Electromagnetic Systems and EMI Mitigation for SKA there. He has held a number of visiting appointments, including at the University of 
Arizona; Cambridge University, U.K.; Delft University of Technology, The Netherlands; and the University of Manchester, U.K. His main research interest through most of his career has been computational electromagnetics (CEM), and he has published extensively on this topic. He is the author of Computational Electromagnetics for $R F$ and Microwave Engineering (Cambridge Univ. Press, 1st ed, 2005, 2nd ed., 2011). Recently, he his interests have expanded to include engineering electromagnetics for radio astronomy.

Prof. Davidson is a member of the South African Institute of Electrical Engineers and the Applied Computational Electromagnetic Society. He was a recipient of the South African FRD (now NRF) President's Award in 1996. He received the Rector's Award for Excellent Research from the University of Stellenbosch in 2005 . He has been actively involved with various IEEE activities; currently, he is the editor of the "EM Programmer's Notebook" column of the IEEE Antennas And Propagation Magazine, and serves on the IEEE Antennas and Propagation AdCom (2011-2013). He was Chair of the local organizing committee of ICEAA'12-IEEE APWC-EEIS'12, held in Cape Town in September 2012

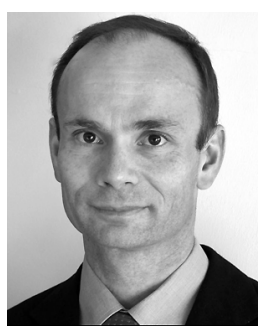

Ulrich Jakobus (F'13) received the Dipl.-Ing. and Dr.-Ing. degrees in electrical engineering from the University of Stuttgart, Stuttgart, Germany, in 1991 and 1994, respectively, and the Habilitation in 1999 and became Privatdozent for Radio Frequency Technology at the same university.

From 1991 to 2000, he was with the Institut für Hochfrequenztechnik, University of Stuttgart. There his main areas of research included numerical techniques in electromagnetics, antennas, electromagnetic compatibility, and bioelectromagnetics. Of particular interest were hybrid methods combining the method of moments with high-frequency asymptotic techniques and this research laid the foundations for the commercial electromagnetics code FEKO. Since 2000, he has been Director of EM Software and Systems-S.A. (Pty) Ltd, Stellenbosch, South Africa, and since 2002 also of EM Software and Systems GmbH, Böblingen, Germany, focusing on the development of FEKO as FEKO Product Manager. He has published extensively on computational electromagnetics and related topics.

Dr. Jakobus received several awards for his research, including the ACES 1996 Outstanding Paper Award and the Heinz-Maier Leibnitz prize 1998 by the Deutsche Forschungsgemeinschaft. He is a member of URSI commission B, the IEEE (elected to the grade of Fellow in 2013), the German VDE/ITG, and a member of the Applied Computational Electromagnetics Society (ACES), where he was also elected Fellow in 2013 and currently serves on the Board of Directors.

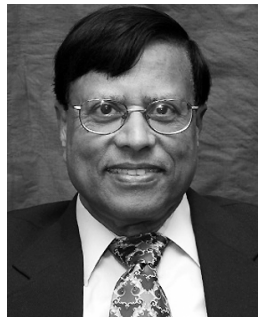

Raj Mittra (LF'96) is a Professor in the Electrical Engineering Department, Pennsylvania State University, State College, PA, where he is the Director of the Electromagnetic Communication Laboratory. $\mathrm{He}$ is also President of RM Associates, a consulting company founded in 1980, which provides services to industrial and governmental organizations, both in the U.S. and abroad. He has published over 1000 technical papers and more than 30 book chapters on various topics related to electromagnetics, antennas, microwaves, and electronic packaging. He has advised more than $100 \mathrm{Ph} . \mathrm{D}$. and an equal number of M.S. thesis students over the years, and has also mentored more than 50 postdocs.

Prof. Mittra has numerous IEEE awards to his credit.

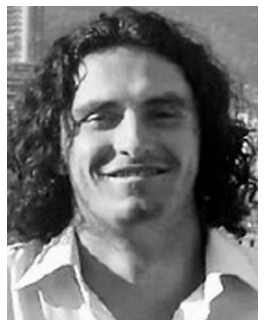

Dirk de Villiers (S'05-M'08) was born in Langebaan, South Africa, on October 13, 1982. He received the B.Eng. and Ph.D. degrees in electrical and electronic engineering from the University of Stellenbosch, Stellenbosch, South Africa, in 2004 and 2007, respectively.

From 2005 to 2007 , he spent several months as a Visiting Researcher with the Computational Modeling and Programming Group at the University of Antwerp, Antwerp, Belgium. From 2008 to 2009, he was a Post-Doctoral Fellow at the University of Stellenbosch working on antenna feeds for the South African SKA program. During this time he was also a part-time Lecturer at the Cape Peninsula University of Technology. He is currently a Senior Lecturer at the University of Stellenbosch, and his main research interests include reflector antennas as well as the design of wide band microwave components such as combiners, filters, and antennas. 